\title{
Aortic Arch Redo Surgery for Endograft Migration and Premature Thrombosis of Debranching Graft
}

\author{
Yihua Liu $^{1}$ Pablo Maureira ${ }^{1} \quad$ Thiery Folliguet $^{1} \quad$ Jean-Pierre Villemot ${ }^{1}$ \\ ${ }^{1}$ Department of Cardiovascular Surgery and Heart Transplantation, \\ University of Lorraine, Vandoeuvre-Les-Nancy, France \\ Thorac Cardiovasc Surg Rep 2013;2:53-56.

\begin{abstract}
Address for correspondence Pablo Maureira, MD, PhD, Department of Cardiovascular Surgery and Heart Transplantation, 1, Alle Du Morvan Chu, Brabois, Vandoeuvre-Les-Nancy 54500, France (e-mail: pablomaureira@hotmail.com).
\end{abstract}

\begin{abstract}
Background Thoracic endovascular aortic repair (TEVAR) has become the treatment of choice in the management of the aortic arch and descending aorta diseases. Thrombosis is a common reason for vascular graft failure, but there is no consensus on the anticoagulation management after placement of vascular graft.

Keywords

- aortic disease

- coagulation/ anticoagulation

- endovascular procedures/stents

Case Description A 21-year-old patient with traumatic rupture of aortic isthmus underwent redo open surgery for two successive complications: stent-graft migration and premature debranching prosthesis thrombosis.

Conclusion Open surgery remains an efficient approach when TEVAR is contraindicated or failed. Postoperatively pharmacological prophylaxis against vascular grafts' thrombosis should be emphasized.
\end{abstract}

\section{Introduction}

This case report aims to report our experience in the management of two successive major complications in a young patient after endovascular intervention for traumatic rupture of aortic isthmus: cranial migration of the endovascular stent graft that imposed an immediate conversion to open surgery and a premature thrombosis of the debranching graft that required redo surgery to reconstruct aortic arch. We discuss thereafter the role of open surgery in the treatment of aortic isthmus rupture, the prevention and follow-up against graft thrombosis. Meanwhile, we described our technique to minimize deep hypothermia circulatory arrest time in aortic arch surgery.

\section{Case Description}

In April 2011, a 21-year-old man met with a traffic accident that resulted in a polytrauma, specifically left diaphragmatic rupture with intestinal hernia, intrathoracic spleen rupture, hepatic and pancreatic contusion, multiple fracture, and aortic isthmus rupture. After general and orthopedic emer- gency operation in a local hospital, the patient was transferred to our center for the management of traumatic rupture of the aortic isthmus. After precise sizing, we decided to implant an endovascular stent graft (Medtronic Valiant, Medtronic Inc., Minneapolis, United States, $24 \mathrm{~mm} \times 10 \mathrm{~cm}$ ) through femoral artery in hybrid operating room. To achieve a safe landing zone ( $>20 \mathrm{~mm}$ ), we have planned to occlude the left subclavian artery. However, during deployment of the endovascular graft, the stent graft migrated cranially into the zone " 0 ," which was confirmed by perioperative angiography; fortunately, the supra-aortic vessels were permeable because of a type I endoleak. We immediately converted to open surgery to transpose the supra-aortic vessels with a bifurcated vascular graft (Intergard-K 14/7, Maquet Getinge Group, Rastatt, Germany). The operation was performed via sternotomy without cardiopulmonary bypass (CPB). The stent graft was not retrieved because the distal extremity covered well the ruptured portion. After transposition procedure, the proximal end of endograft was sealed by balloon inflation. The recovery was uneventful. The patient presented no neurological deficits, or difficulty in moving the left upper arm. received

May 4, 2013

accepted after revision

June 24, 2013

published online

September 10, 2013
DOI http://dx.doi.org/

10.1055/s-0033-1351356. ISSN 2194-7635. (c) 2013 Georg Thieme Verlag KG
Stuttgart · New York

License terms

(1) $\circledast$ 


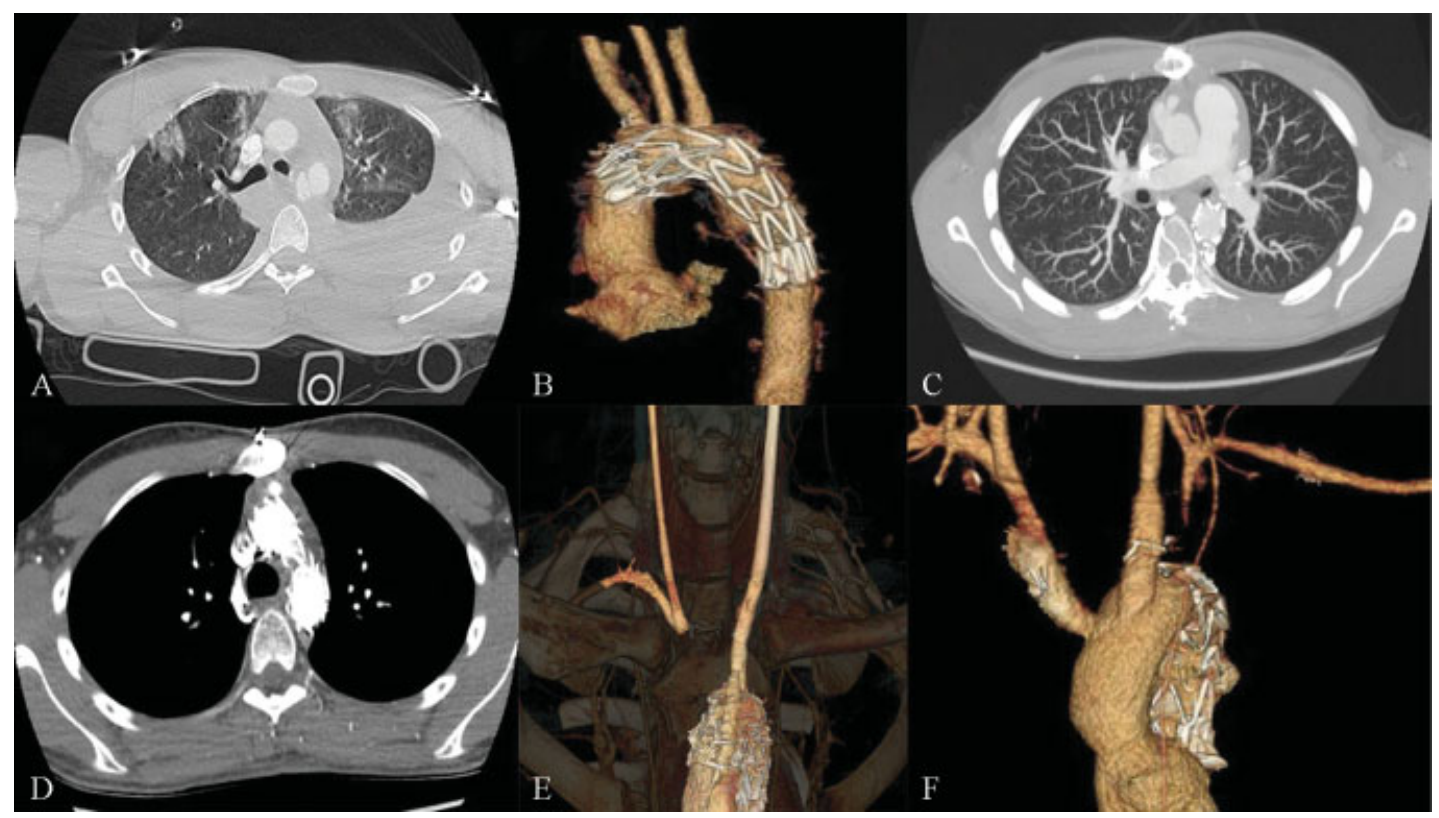

Fig. 1 Imaging manifestations and disease evolutions. (A) Traumatic rupture of aortic isthmus; (B) reconstructed angiography in hybrid operating room showed endovascular graft overstenting supra-aortic vessels; (C) CTA of control 3 months later showed permeable debranching graft; ( $D$ and $E$ ) one branch of graft on the side of innominate artery was thrombosed; and (F) CTA after redo surgery demonstrated permeable grafts. CTA, computed tomography angiography.

During follow-up, the patient did well in ordinary life as well as in his studies. The control imaging exams in 6 months and 1 year were normal. However, 15 months later, he manifested several periods of horizontal diplopia and one episode of transient paresia of the right arm. The computed tomography angiography (CTA) showed threatening thrombosis in the debranching graft. The patient was immediately hospitalized for an emergent redo surgery. In this redo surgery, we removed the thrombosed debranching graft as well as part of ascending aorta and stent graft; we reconstructed the aortic arch with a polyester vascular graft (Intergard 26) and two Dacron (polyethylene terephthalate) grafts $(8 \mathrm{~mm})$. The redo operation was uneventful and the recovery was favorable. The patient was transferred to the ordinary ward on the third day and discharged on the eighth day. The CTA and Echo-Doppler before discharge showed permeable vascular prosthesis without complications. To date, the patient is well and will be followed regularly in the outpatient clinic (-Figs. $\mathbf{1}$ and $\mathbf{2}$ ).

\section{Discussion}

Since two decades, thoracic endovascular aortic repair (TEVAR) has emerged as an alternative of traditional open surgery for the treatment of traumatic aortic injury (TAI). The short-term and midterm outcomes were in favor of TEVAR compared with open repair considering their invasiveness and postoperative complications. ${ }^{1}$ However, open surgery remains an efficient approach in the management of TAI and should not be ignored in the era of TEVAR. First, unlike open surgery, TEVAR lacks long-term follow-up outcomes to substantiate its effectiveness and durability in the treatment of TAI. The late complications of TEVAR such as secondary endoleak, device failure, and endograft migration remain a considerable issue and require yearly imaging exams (contrast-enhanced CT, magnetic resonance imaging, or angiography). Second, in some clinical and anatomical challenging situations, such as instable hemodynamics requiring $\mathrm{CPB}$, acute aortic arch angulations, great tortuosity of the aorta, and a small aortic diameter especially in the young patient, TEVAR is not considered to be suitable. ${ }^{2}$ Third, it is advisable to perform TEVAR with standing by instruments and equip for conversion to open surgery. Once the periprocedural complications occur such as graft malposition, migration, collapse, and primary endoleak, conversion to open repair is still an efficient salvaging technique.

As well as its extensive use in the management of traumatic aortic injuries, TEVAR in association with supra-aortic vessels transposition technique becomes an alternative of traditional elephant trunk procedure in the management of aortic arch diseases such as aneurysm and type B aortic dissection. The disadvantages with open surgery are significantly high perioperative mortality and complication that result majorly from long application of CPB and deep hypothermic circulatory arrest (DHCA). During this redo surgery, we have worked out a simple but useful approach to minimize the CPB and DHCA time. This technique consists of femorofemoral CPB, selective antegrade cerebral perfusion, retrograde infusion of cardioplegic solution, and "endoclamping" descending aorta with an inflated 20-F Foley catheter. The femoral CPB and antegrade cerebral perfusion were established before sternotomy. The patient was cooled to $25^{\circ} \mathrm{C}$ when we dissected the adherence and removed the 


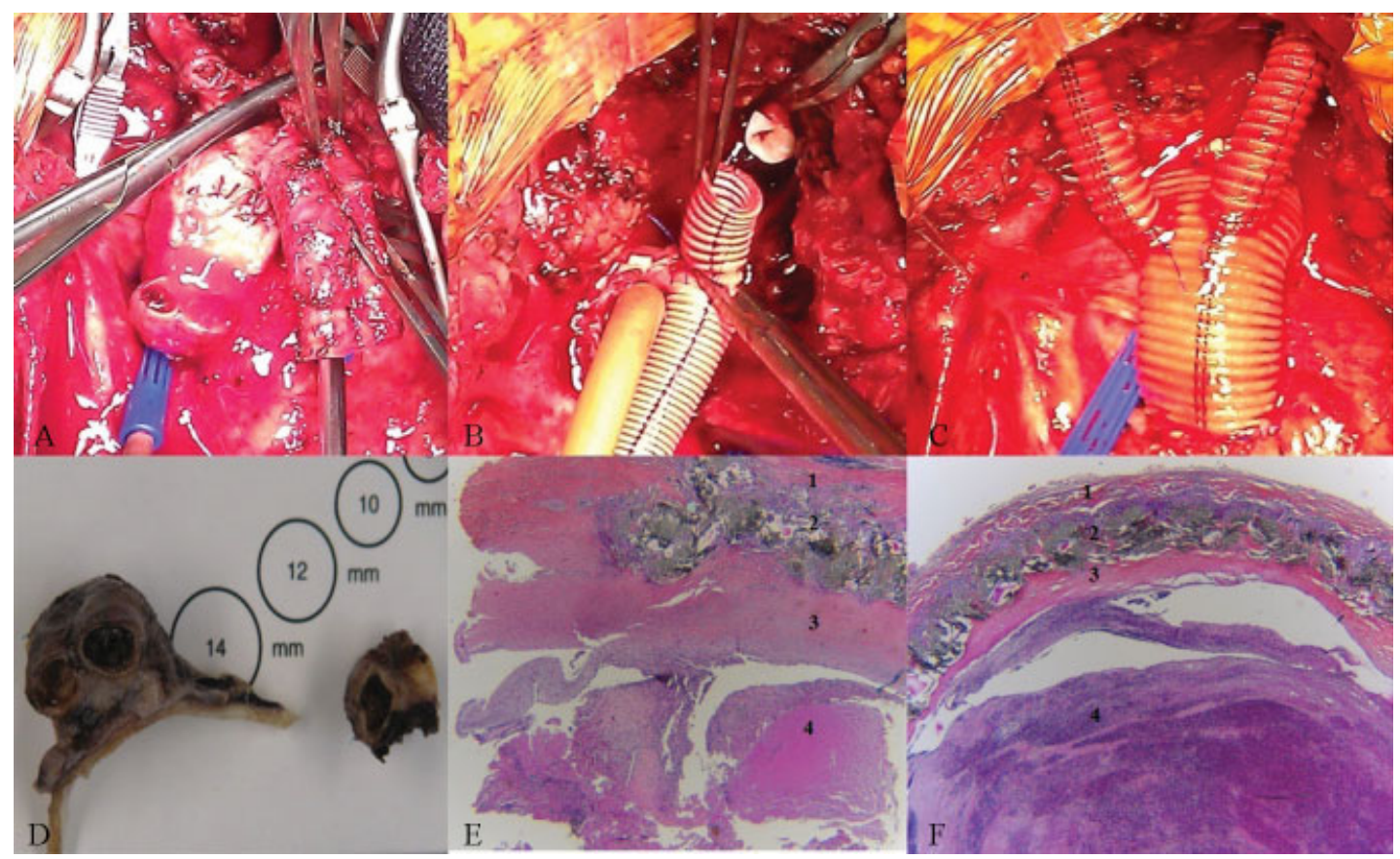

Fig. 2 (A) Thrombus in the debranching graft; (B) endoclamping with an inflated Foley catheter in the descending thoracic aorta ensured a dry operating field when performing anastomosis; (C) reconstruction of aortic arch with a 26-mm Intergard graft and two 8-mm Dacron grafts; (D) macroscopic view of histopathology, left and right piece corresponded proximal and distal portions of thrombosed graft; hematoxylin-eosin stained sections $(\times 10)$ of proximal portion $(E)$ and distal portion $(F)$ showed identical structures: 1 . artery wall, aorta $(E)$ and innominate artery $(F)$; 2. synthetic vascular graft; 3. neointima hyperplasia; 4. organized (E) or newly formed (F) thrombus.

debranching graft. The CPB was temporarily ceased when we transected the aortic arch and the Foley catheter was inserted into the descending aorta and inflated with $20 \mathrm{~mL}$ sterile physiological saline to "endoclamp" the aorta. The CPB was then restarted when we performed the distal anastomosis. Once distal anastomosis was finished, the Foley catheter was retrieved and replaced by a traditional aortic clamp, we continued other anastomosis when the patient was warmed. This technique allowed us to perform aortic arch reconstruction with mild hypothermia $\left(25^{\circ} \mathrm{C}\right)$, a dry operating field, short DHCA time (12 $\mathrm{min}$ ), and acceptable CPB time (138 min). The outcomes were satisfactory: quick recovery and short hospital stay.

Thrombosis is a common reason for premature or delayed synthetic vascular graft failure. The incidence of graft thrombosis varies from 3 to $19 \%$ and the prognosis ranges from spontaneous resolution to complete thrombosis. ${ }^{3}$ The histological basis of graft thrombosis is neointimal hyperplasia nearby anastomosis and absence of endothelial cells, which are natural anticoagulant agents. ${ }^{4}$ The treatments of graft thrombosis include thrombolysis and surgical replacement of thrombosed graft. The pharmacological prophylaxis against graft thrombosis formation is more efficient and regular imaging follow-up with CTA for early diagnosis is essential, especially in the patients with risk factors such as small caliber graft, hypercoagulative state. To date, no guideline of postoperative anticoagulation management after placement of vascular graft is available. Daily ingestion of moderate dose of aspirin is often prac- ticed. In this young patient with low-risk factors of thrombosis formation, no anticoagulant medication was prescribed after his first discharge; 15 months later, he suffered a serious graft thrombosis disease and underwent a complicated redo surgery. Thus, we propose to enlarge the indication of pharmacological prophylaxis against postoperative graft thrombosis even in the low-risk patients to improve grafts' long-term patency, and to practice regular clinical and imaging follow-up for early diagnosis and intervention.

\section{Conclusion}

Endovascular repair becomes a treatment of choice for traumatic rupture of aortic isthmus. However, open surgery remains a notable approach, especially in the management of immediate and late complications of endovascular procedures. After supra-aortic vessel debranching surgery, meticulous clinical and precise imaging follow-up are mandatory, and pharmacological prophylaxis against graft thrombosis should be considered even in patients with low thrombosis risks.

\section{References}

1 Mosquera VX, Marini M, Lopez-Perez JM, et al. Role of conservative management in traumatic aortic injury: comparison of long-term results of conservative, surgical, and endovascular treatment. J Thorac Cardiovasc Surg 2011;142(3):614-621 
56 Aortic Arch Redo Surgery Liu et al.

2 Lee WA, Matsumura JS, Mitchell RS, et al. Endovascular repair of traumatic thoracic aortic injury: clinical practice guidelines of the Society for Vascular Surgery. J Vasc Surg 2011;53(1):187-192

3 Mehta RI, Mukherjee AK, Patterson TD, Fishbein MC. Pathology of explanted polytetrafluoroethylene vascular grafts. Cardiovasc Pathol 2011;20(4):213-221
4 Scharn DM, Daamen WF, van Kuppevelt TH, van der Vliet JA. Biological mechanisms influencing prosthetic bypass graft patency: possible targets for modern graft design. Eur J Vasc Endovasc Surg 2012;43(1):66-72 